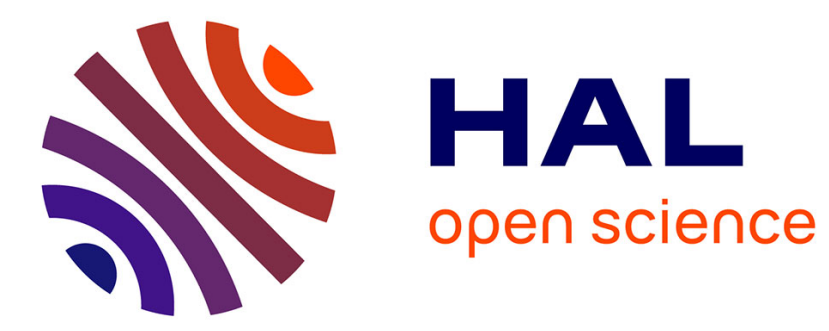

\title{
Usual Anderson localization restored in bilayered left- and right-handed structures
}

Agnès Maurel, Abdelwaheb Ourir, Jean-François Mercier, Vincent Pagneux

\section{To cite this version:}

Agnès Maurel, Abdelwaheb Ourir, Jean-François Mercier, Vincent Pagneux. Usual Anderson localization restored in bilayered left- and right-handed structures. Physical Review B: Condensed Matter and Materials Physics (1998-2015), 2012, 85 (20), 10.1103/physrevb.85.205138 . hal-00849562

HAL Id: hal-00849562

https://hal-ensta-paris.archives-ouvertes.fr/hal-00849562

Submitted on 26 Nov 2021

HAL is a multi-disciplinary open access archive for the deposit and dissemination of scientific research documents, whether they are published or not. The documents may come from teaching and research institutions in France or abroad, or from public or private research centers.
L'archive ouverte pluridisciplinaire HAL, est destinée au dépôt et à la diffusion de documents scientifiques de niveau recherche, publiés ou non, émanant des établissements d'enseignement et de recherche français ou étrangers, des laboratoires publics ou privés. 


\title{
Usual Anderson localization restored in bilayered left- and right-handed structures
}

\author{
Agnès Maurel and Abdelwaheb Ourir \\ LOA/Institut Langevin, ESPCI, UMR CNRS 7587, 10 rue Vauquelin, 75005 Paris, France \\ Jean-François Mercier \\ Laboratoire Poems, ENSTA, UMR CNRS 7231, 32 bd Victor, 75015 Paris, France \\ Vincent Pagneux \\ LAUM, Université du Maine, UMR CNRS 6613, Avenue Olivier Messiaen, 72085 Le Mans, France
}

(Received 30 December 2011; revised manuscript received 3 March 2012; published 25 May 2012)

\begin{abstract}
We present a study of the attenuation length in a one-dimensional array of alternating left- and right-handed materials in which both the permittivities and the permeabilities are disordered. This type of structure has been shown to present an anomaly in the attenuation length when only permeabilities are disordered. We derive a simple analytical expression of the attenuation length, when the disorder in the refraction index is due to perturbations in both the permeability and the permittivity. Our expression is able to explain the transition to the anomalous behavior when perturbation only in the permeability or only in the permittivity is considered. Besides, we show that the anomaly is dramatically affected when considering perturbations in permeability and permittivity. The coupling effects are able to restore the ordinary localization length.
\end{abstract}

DOI: 10.1103/PhysRevB.85.205138

PACS number(s): 42.25.Fx, 72.15.Rn

\section{INTRODUCTION}

In electromagnetism, the design of materials with negative index has offered new perspectives in nanoscience. ${ }^{1,2}$ In light of the properties of these new materials, the propagation and localization in a periodic one-dimensional (1D) system has been revisited (e.g., Ref. 3; see also Refs. 4 and 5 for a review on 1D propagation in disordered media). Recently, periodic bilayered structures formed by alternating left-handed and righthanded materials have been investigated and they revealed the surprising behavior of the so-called mixed stack. ${ }^{6,7}$ A mixed stack is a particular periodic structure with a unit cell formed of two layers of same length $d=d_{1}=d_{2}$ and opposite refractive indices $n_{1}=-n_{2}$. Such unit cell does not accumulate phase and leads to a transparent structure with perfect transmission. When perturbation in the permittivity is introduced, leading to perturbed refractive index and perturbed impedance, an anomaly in the attenuation length has been exhibited, resulting in a significant suppression of Anderson localization. ${ }^{6,7}$ Very recently, the localization length $L_{\text {loc }}$ has been derived for this case, ${ }^{8}$ leading to the prediction $L_{\mathrm{loc}} \propto Q^{-4}(k d)^{-8}$ ( $Q$ being the perturbation strength and $k=2 \pi / \lambda$ the wave number) which is valid in the low-frequency regime. In this paper, we revisit this anomaly. By deriving a simple analytical expression for the attenuation length in mixed stacks, we can analyze the conditions leading to the transition to the anomalous behavior. We show that the transition begins for $N Q^{2}(k d)^{2}=1$ and that increasing the length $N$ of the structure produces an increase in the attenuation length until it reaches the localization length as $N \rightarrow \infty$. For $\lambda / d \lesssim 10$, the localization length is reached after $N Q^{2}(k d)^{2}>100$ and our analytical expression is accurate to characterize the transition. For $\lambda / d \gtrsim 10$, at lower frequency, we may notice in the numerical results of Refs. 7 and 8 that the localization length is reached after $N Q^{4}(k d)^{8}>100$, that is, for much larger structure sizes (and this is the regime predicted in Ref. 8). Of course, this raises the question of whether or not such regimes can take place in the experiments involving actual designed structures. Currently, designed metamaterial layers work at typically $\lambda \sim d{ }^{9,10}$ This is the case because such layers are realized by stacking two-dimensional arrays of resonators of thickness $\sim \lambda / 10$ (for instance, split ring resonators combined with continuous wires, ${ }^{9}$ or fishnets ${ }^{10}$ ) and, to limit the losses, a small number (about 10) of stacks is used. In optics, the problem of losses in metal is crucial and the most recent metamaterial layers have micrometer lengths in the infrared frequency regime. ${ }^{10}$ For the application to bilayered structures using left-handed materials, the problem of losses remains, in addition to compactness constraints, which limits also the value of $N$. Under these conditions, it is of importance to carefully describe the transmission properties of structures with moderate size and working in the frequency regime $\lambda \sim d$.

Another important practical aspect is the fact that a perturbation in the refractive index results in general from simultaneous perturbations in permittivity $\epsilon$ and in permeability $\mu$. These perturbations can be evaluated but they cannot be controlled, in particular at microscales and nanoscales because of manufacturing inaccuracies. In this paper, we show that the behaviors of the attenuation and localization lengths are dramatically different when including a perturbation in the permeability $\delta \mu$ in addition to the previously considered perturbation in permittivity $\delta \epsilon$ (an effect recently observed in Ref. 11). Although the resulting perturbation in the refractive index simply follows from superposition, $\delta n=\delta \mu+\delta \epsilon$ at dominant order, the obtained attenuation length does not follow from superposition. Indeed, coupling effects in $\epsilon$ and $\mu$ are able to make the anomaly disappear, or in other words, coupling effects restore the ordinary localization.

\section{PERTURBATIVE EXPANSION}

We propose a derivation of the attenuation length $l_{N}$ (with $1 / l_{N} \equiv-\left\langle\ln \left|T_{N}\right|\right\rangle / N$ and $T_{N}$ is the transmission coefficient) 


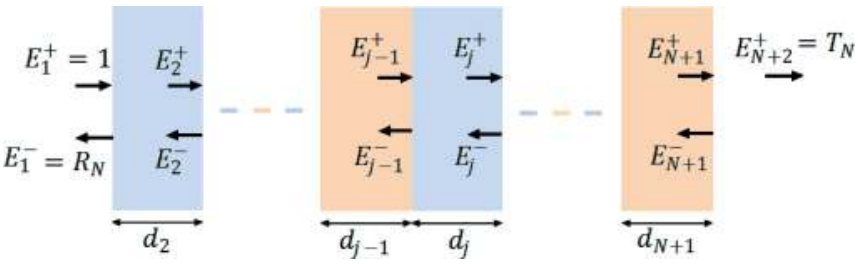

FIG. 1. (Color online) Geometry of the layered structure. The electric $E_{j}$ and magnetic $H_{j}$ fields are parallel to the interfaces.

based on the analysis of the electric and magnetic fields $E, H$ in a structure of length $N\left(d_{1}+d_{2}\right) / 2$, with $N / 2$ unit cells ( $N$ is an even number) (see Fig. 1). In the unperturbed structure, the unit cell is formed of two layers of lengths $d_{a}$ $(a=1,2)$, with refractive indices $n_{a}= \pm \sqrt{\epsilon_{a} \mu_{a}}$ (the upper and lower signs, respectively, refer to right-handed and lefthanded materials). The unperturbed structure is transparent when $Z_{1}=Z_{2}$, with $Z_{a}=Z_{0} \sqrt{\mu_{a} / \epsilon_{a}}$ (perfect impedance matching without reflection). In each layer $j$, perturbations in both $\epsilon$ and $\mu$ are considered, $\mu_{j}=\mu_{a}\left(1+\delta \mu_{j}\right)^{2}$ and $\epsilon_{j}=\epsilon_{a}\left(1+\delta \epsilon_{j}\right)^{2}$ with uncorrelated $\delta \mu_{j}$ and $\delta \epsilon_{j}$, distributed uniformly on $\left[-Q_{\mu}, Q_{\mu}\right]$ and $\left[-Q_{\epsilon}, Q_{\epsilon}\right]$, respectively. The resulting perturbation in the refractive index $\delta n_{j}$ is distributed on $[-Q, Q]$ with $Q=\sqrt{Q_{\epsilon}^{2}+Q_{\mu}^{2}}$.

We define the electric and magnetic fields $E_{j}$ and $H_{j}$ at the boundary between the layers $j$ and $(j+1)$, satisfying, for $j=2, \ldots N+2$,

$$
\left(\begin{array}{l}
E_{j-1} \\
H_{j-1}
\end{array}\right)=\left(\begin{array}{lc}
\cos \varphi_{j} & i Z_{j} \sin \varphi_{j} \\
i \sin \varphi_{j} / Z_{j} & \cos \varphi_{j}
\end{array}\right)\left(\begin{array}{l}
E_{j} \\
H_{j}
\end{array}\right)
$$

with $\varphi_{j} \equiv k_{j} d_{j}$ and where the time dependence $e^{i \omega t}$ has been considered. For small disorder, the impedances remain close to each other and we introduce a small parameter $\delta_{j} \equiv\left(Z_{j} / Z_{j-1}-1\right) / 2$. Decomposing $E_{j}$ into right-going $E_{j}^{+}$ and left-going $E_{j}^{-}$waves, we get

$$
\begin{aligned}
e^{i \varphi_{j}} E_{j}^{+} & =E_{j-1}^{+}+\delta_{j}\left(E_{j-1}^{+}-E_{j-1}^{-}\right), \\
e^{-i \varphi_{j}} E_{j}^{-} & =E_{j-1}^{-}-\delta_{j}\left(E_{j-1}^{+}-E_{j-1}^{-}\right),
\end{aligned}
$$

with the boundary conditions $E_{1}^{+}=1, E_{N+2}^{+}=T_{N}, E_{1}^{-}=R$, $E_{N+2}^{-}=0$. Defining a term of phase accumulation $\phi_{j} \equiv$ $\sum_{l=2}^{j} \varphi_{l}$, and the quantities $P_{j} \equiv e^{i \phi_{j}} E_{j}^{+}$and $D_{j} \equiv e^{-i \phi_{j}} E_{j}^{-}$, we obtain

$$
\begin{aligned}
& P_{j}=1+\sum_{l=2}^{j} \delta_{l}\left[P_{l-1}-e^{2 i \phi_{l-1}} D_{l-1}\right], \quad 2 \leqslant j \leqslant N+2, \\
& D_{j}=\sum_{l=j+1}^{N+2} \delta_{l}\left[e^{-2 i \phi_{l-1}} P_{l-1}-D_{l-1}\right], \quad 1 \leqslant j \leqslant N+1,
\end{aligned}
$$

where we used $P_{1}=1$ and $D_{N+2}=0$. The transmission coefficient is $T_{N}=e^{-i \phi_{N+1}} P_{N+2}$ and the aim is to derive the attenuation length $l_{N}$. This is achieved by solving (3) iteratively owing to the small parameter $\delta_{j}$. At the second iteration,

$$
\begin{aligned}
T_{N} e^{i \phi_{N+1}}= & 1+\underbrace{\sum_{n=2}^{N+2} \delta_{n}}_{S_{1}} \\
& +\underbrace{\sum_{n=2}^{N+2} \sum_{m=2}^{n-1} \delta_{n} \delta_{m}-\sum_{n=2}^{N+2} \sum_{m=n}^{N+2} \delta_{n} \delta_{m} e^{2 i\left(\phi_{n-1}-\phi_{m-1}\right)}}_{S_{2}} \\
& +O\left(Q^{3}\right),
\end{aligned}
$$

and $\quad \delta_{n}=1 / 2\left[\left(\delta \mu_{n}-\delta \epsilon_{n}\right)\left(1-\delta \epsilon_{n}\right)-\left(\delta \mu_{n-1}-\delta \epsilon_{n-1}\right)(1-\right.$ $\left.\left.\delta \mu_{n-1}\right)-\left(\delta \mu_{n}-\delta \epsilon_{n}\right)\left(\delta \mu_{n-1}-\delta \epsilon_{n-1}\right)\right]+O\left(Q^{3}\right)$. We have

$$
l_{N}^{-1}=-\frac{1}{N} \operatorname{Re}\left(\left\langle S_{1}+S_{2}\right\rangle\right)+O\left(Q^{3}\right),
$$

where we have used $\left\langle\left|S_{1}\right|^{2}\right\rangle=O\left(Q^{3}\right)$. Analytical expressions presented in this paper are obtained at the second iteration, leading to a prediction accurate up to $O\left(Q^{3}\right)$. Note that this second order in $Q$ is also the approximation used in the semianalytical WSA used in Refs. 6 and 7-see Eq. (12) in Ref. 7 where the term $R_{n-1}^{2} t_{n}^{2} r_{n}$ is omitted [in Ref. 7, $\left(r_{n}, t_{n}\right)$ are the reflection and transmission coefficients of a single layer], and this term is $O\left(Q^{3}\right)$ at best. The third-order calculation is presented in Appendix A. It is found to vanish when the second-order vanishes (namely, the asymptotic $N \rightarrow \infty$ and the low-frequency regime $Q k d \ll 1$ ), and it is thus unable to capture the behavior of the localization length when the anomaly occurs. This is consistent with the prediction in Ref. 8, where the localization length is found to vary as $Q^{-4}$ in the low-frequency regime.

In the following, for clarity, we first inspect the case where only a perturbation in $\epsilon$ is considered, and then, we inspect the case with perturbations both in $\epsilon$ and $\mu$ for a unit cell with no phase accumulation.

\section{LAYERS WITH PERTURBATIONS ONLY IN $\epsilon$}

We first consider the case where $Q_{\mu}=0$ and $Q=Q_{\epsilon}$, corresponding to the situation considered in Refs. 6-8. We can notice that the result is the same if $Q=Q_{\mu}, Q_{\epsilon}=0$. The attenuation length for a structure of size $N\left(d_{1}+d_{2}\right) / 2$ is

$$
\begin{gathered}
\frac{1}{l_{N}}=\frac{1}{l_{1}}-\frac{Q^{2}}{12} \mathcal{A}\left(k_{1} d_{1}, k_{2} d_{2}, Q, N\right), \\
\frac{1}{l_{1}}=\frac{Q^{2}}{12}\left\{1-\frac{1}{2}\left[f\left(x_{1}\right) \cos 2 k_{1} d_{1}+f\left(x_{2}\right) \cos 2 k_{2} d_{2}\right]\right\}, \\
\text { with } x_{a} \equiv 2 Q k_{a} d_{a}(a=1,2) \text { and } \\
f(x) \equiv 3 \operatorname{sinc} x-6(\operatorname{sinc} x-\cos x) / x^{2},
\end{gathered}
$$

where $\operatorname{sinc} x \equiv \sin x / x$. The general form of $\mathcal{A}$ has no particular interest and we refer to Appendix A for its expression and its asymptotic behavior (simplified expressions are given in the following).

The key point is the following: $\mathcal{A}$ depends on $N$, and it is responsible for the anomaly that occurs only for $k_{2} d_{2}=-k_{1} d_{1}$ (this agrees with the observation of Ref. 12 that a structure, 


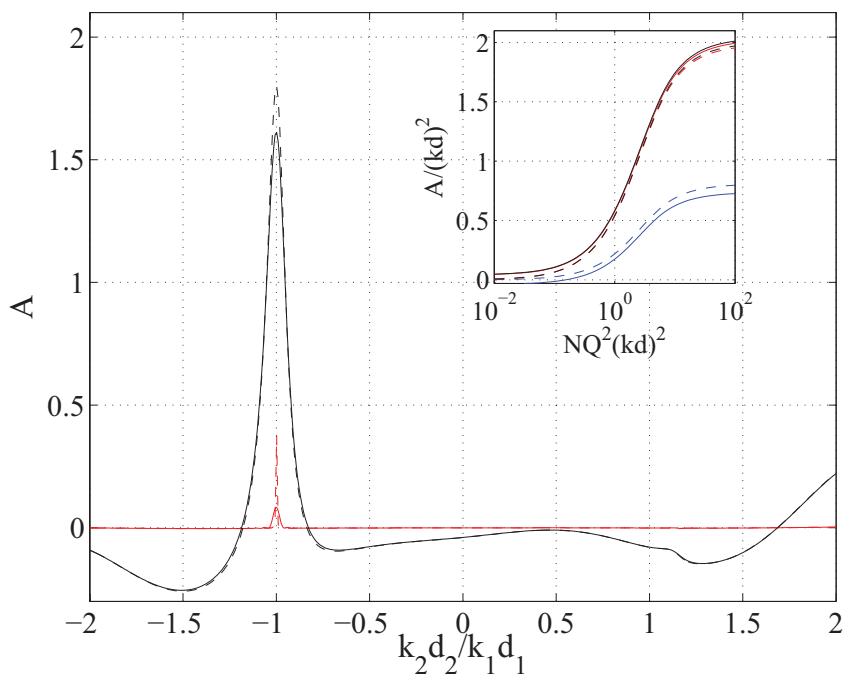

FIG. 2. (Color online) $\mathcal{A}$ as a function of $k_{2} d_{2} / k_{1} d_{1}$, curves given for $k_{1} d_{1}=2 \pi / 4.3$ : lower red curve $Q=0.025$ and upper black curve $Q=0.25$ (plain line $N=100$ and dotted line $N=1000$ ). The inset shows $\mathcal{A}$ as a function of $N$, for $k d=k_{1} d_{1}=-k_{2} d_{2} . N$ is normalized to $N Q^{2}(k d)^{2}$ and $\mathcal{A}$ is normalized to $\mathcal{A} /(k d)^{2}$. $\lambda / d=4$ (blue lower curve), and $\lambda / d=40,400$ (upper red and black curves, almost undistinguishable) for $Q=0.025$ (plain line) and $Q=0.25$ (dotted line).

with $d_{1} \neq d_{2}$ and $k_{2}=-k_{1}$, does not present the anomaly). This is illustrated in Fig. 2: it can be seen that $\mathcal{A}$ has amplitude unity only in the vicinity of $k_{2} d_{2}=-k_{1} d_{1}$; otherwise it is at least one order of magnitude smaller. In this latter case, it is negligible and the attenuation length takes a simple form, independent of $N, l_{N}=l_{1}$ that characterizes the transmission properties of the structure for any size $N$. Moreover, we recover the expected behavior of the localization length in the highfrequency regime, $l_{1} \rightarrow 12 / Q^{2}$ since $f \rightarrow 0$, and in the lowfrequency regime, $1 / l_{1}=Q^{2} / 12\left(\sin ^{2} k_{1} d_{1}+\sin ^{2} k_{2} d_{2}\right)$ since $f \rightarrow 1$, as obtained in Ref. 12. Of course, taking into account the exact form of the function $f$ allows us to better describe the attenuation length for intermediate values of the wavelength $\lambda$.

If the unit cell does not accumulate phase, for alternating right-handed and left-handed materials with $k d=k_{1} d_{1}=-k_{2} d_{2}$, Eq. (6) simplifies to

$\frac{1}{l_{N}}=\frac{Q^{2}}{12}\left[1-f(x) \cos 2 k d-h^{2}(x) \mathcal{B}(\operatorname{sinc} x, k d, N)\right]$,

with $x \equiv 2 Q k d$,

$$
h(x) \equiv \sqrt{3}[\operatorname{sinc} x-\cos x] / x
$$

[such that $\mathcal{A}=h^{2}(x) \mathcal{B}$ ] and

$$
\begin{aligned}
\mathcal{B}(r, k d, N) \equiv & \frac{1-r \cos 2 k d}{1-r^{2}} \\
& -\frac{1-r^{N}}{N\left(1-r^{2}\right)^{2}}\left[1+r^{2}-2 r \cos 2 k d\right],
\end{aligned}
$$

and here, $r$ is a dummy variable. The main characteristics of $\mathcal{A}$ are illustrated in the inset of Fig. 2. A transition can be observed for $N Q^{2}(k d)^{2} \sim 1$. For $\lambda / d \lesssim 10, \mathcal{A}$ reaches asymptotically $(N \rightarrow \infty)$ a value that does not compensate the $1 / l_{1}$ term in
Eq. (6). Consequently, $1 / l_{N}$ is of the same order as $1 / l_{1}$ and there is no anomaly. For $\lambda / d \gtrsim 10, \mathcal{A}$ reaches asymptotically a value that compensates the $1 / l_{1}$ term in Eq. (6), leading to an increase in $l_{N}$ and thus to the aforementioned anomaly. The asymptotic of $1 / l_{N}$ for $N \rightarrow \infty$ is clearly

$$
1 / l_{N} \rightarrow \frac{Q^{2}}{12}\left[1-f(x) \cos 2 k d-h^{2}(x) \frac{1-\cos 2 k d \operatorname{sinc} x}{1-\operatorname{sinc}^{2} x}\right] .
$$

When $x \equiv 2 Q k d$ is small, $h(x) \sim x / \sqrt{3}$ [Eq. (10)], leading to $h^{2}(x)(1-\cos 2 k d \operatorname{sinc} x) /\left(1-\operatorname{sinc}^{2} x\right)=(1-\cos 2 k d)+$ $O\left(Q^{2}\right)$ [and $f(x) \rightarrow 1$, Eq. (8)]. This means that the asymptotic $N \rightarrow \infty$ makes the leading order of $1 / l_{N}$ [Eq. (12)] to vanish at small enough frequency $(k d \ll 1 / Q$; see also Appendix A). We show in Appendix B that the contribution to $1 / l_{N}$ at third order in $Q$ also vanishes in the same limits, $k d \ll 1 / Q, N \rightarrow \infty$. This means that the fourth order has to be determined, and this is consistent with the result of Ref. 8 where it is shown that the localization length varies as $1 / Q^{4}$ for large $\lambda$. Finally, note that the wavelengths smaller than the unit-cell length $(\lambda / d<1)$ are not concerned by the anomaly because the term $[1-f(2 Q k d) \cos 2 k d] \rightarrow 1$ while $\mathcal{A}$ remains smaller than 0.1 (the behavior of $\mathcal{A}$ for small wavelengths is not reported here).

\section{MIXED STACK WITH DISORDER IN $\epsilon$ AND $\mu$}

To assess the robustness of the anomaly, we consider now perturbations both in $\epsilon$ and $\mu$ but still under the condition of no phase accumulation in the unit cell, $k d=k_{1} d_{1}=-k_{2} d_{2}$. Using the same perturbation technique, we find a generalized expression of Eq. (9):

$$
\begin{aligned}
\frac{1}{l_{N}}= & \frac{Q_{\epsilon}^{2}}{12}\left[1-f\left(x^{\epsilon}\right) \operatorname{sinc} x^{\mu} \cos 2 k d\right] \\
& +\frac{Q_{\mu}^{2}}{12}\left[1-f\left(x^{\mu}\right) \operatorname{sinc} x^{\epsilon} \cos 2 k d\right] \\
& -\frac{Q_{\epsilon} Q_{\mu}}{6} \cos 2 k d h\left(x^{\epsilon}\right) h\left(x^{\mu}\right) \\
& -\frac{1}{12}\left[Q_{\epsilon} h\left(x^{\epsilon}\right) \operatorname{sinc} x^{\mu}-Q_{\mu} h\left(x^{\mu}\right) \operatorname{sinc} x^{\epsilon}\right]^{2} \\
& \times \mathcal{B}(R, k d, N),
\end{aligned}
$$

with $R \equiv \operatorname{sinc} x^{\epsilon} \operatorname{sinc} x^{\mu}$. Here, the first three terms are independent of $N$ and they essentially follow from the superposition of $l_{1}$ values [ $Q=Q_{\epsilon}$ and $Q_{\mu}$ in Eq. (7)]. The fourth term is dependent on $N$. This term was responsible for the anomaly when $Q_{\mu}=0$, as seen previously, since the asymptotic $N \rightarrow \infty, Q k d \ll 1$ makes $1 / l_{N}$ vanish. Here, the asymptotic leads to

$$
\frac{1}{l_{N}} \rightarrow \frac{Q^{2}}{3}(1-\cos 2 k d) \alpha(1-\alpha),
$$

with $Q^{2} \equiv Q_{\epsilon}^{2}+Q_{\mu}^{2}$ the amplitude of the disorder in the refractive index and $\alpha \equiv Q_{\epsilon}^{2} / Q^{2}$. The leading order in $1 / l_{N}$ vanishes for disorder only in $\epsilon(\alpha=1)$ or for disorder only in $\mu(\alpha=0)$, which produces the anomaly. When perturbations both in $\epsilon$ and $\mu$ are considered, the term does not vanish and 

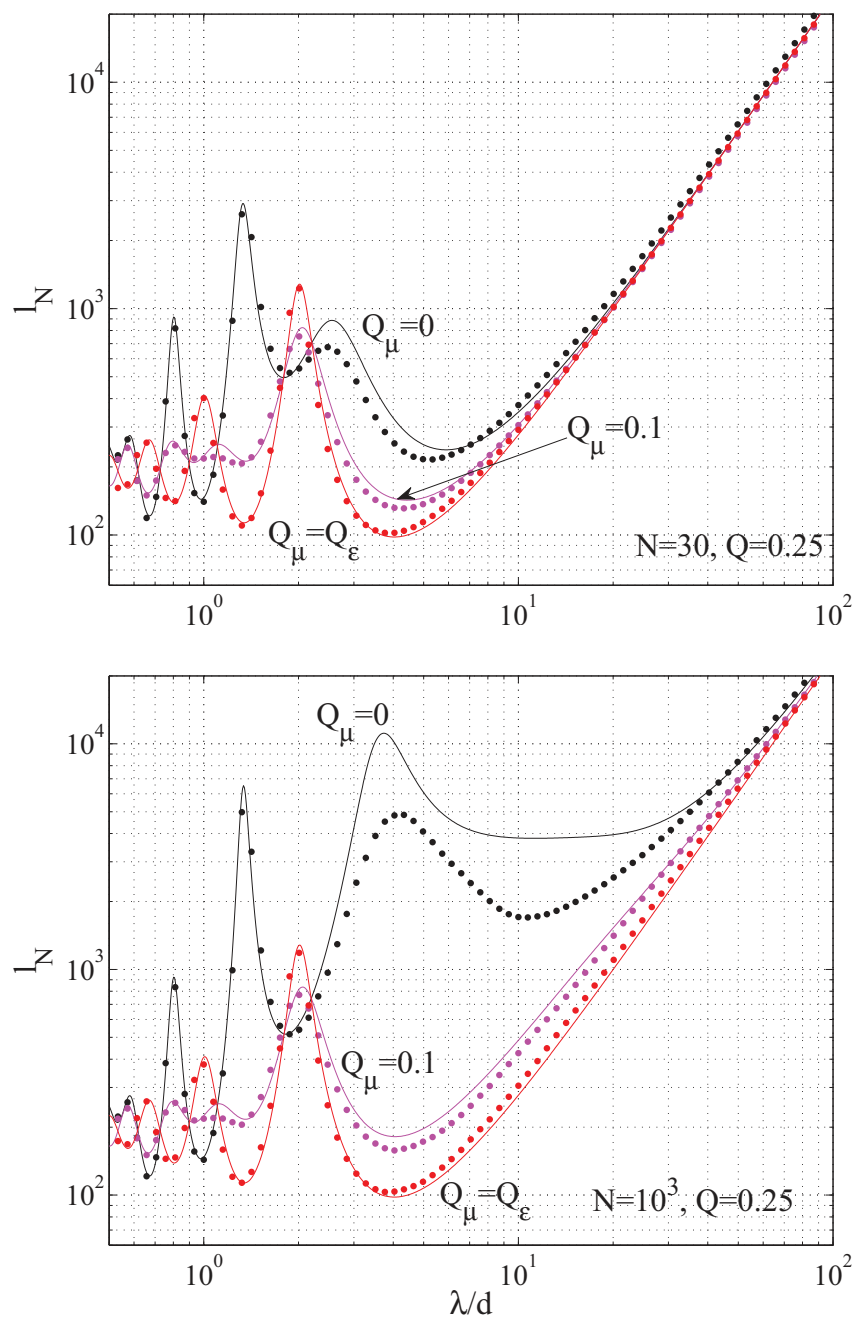

FIG. 3. (Color online) Attenuation length $l_{N}$ of a mixed stack for lengths $N=30$ and $N=10^{3}$ as a function of $\lambda / d$. Dots show the numerical results and plain lines correspond to our prediction in Eq. (13). Different curves correspond to $Q_{\mu}=0$ (black dots), $Q_{\mu}=0.1$ (magenta dots), and $Q_{\mu}=Q_{\epsilon}=Q / \sqrt{2}$ (red dots) for constant $Q=\sqrt{Q_{\mu}^{2}+Q_{\epsilon}^{2}}=0.25$ (constant perturbation strength in the refractive index). Result for the homogeneous stack (usual Anderson localization) are not represented and would be superposed on the $Q_{\epsilon}=Q_{\mu}$ case (red dots).

the leading order remains dominant, which restores the usual Anderson localization.

Our results are exemplified in Figs. 3 and 4 and compared with direct numerical calculations (numerics have been done with $10^{3}-10^{4}$ averages). We considered two different sizes of mixed stacks $N=30$ and $N=10^{3}$ for a constant refractive index perturbation $Q=0.25$, resulting from $Q_{\mu}=0,0.1$ and $Q / \sqrt{2}$ (and $Q_{\epsilon}=\sqrt{Q^{2}-Q_{\mu}^{2}}$ ). Our conclusions are twofold:

(1) When the perturbation in the refractive indices result from perturbations both in $\epsilon$ and $\mu$, a significant decrease in the attenuation length is visible at low frequency (Fig. 3). For $Q_{\mu} \sim Q_{\epsilon}$, the usual localization is restored, similar to the localization observed in the so-called homogeneous stack [corresponding to a unit cell with $k d=k_{1} d_{1}=k_{2} d_{2}$ (Refs. 6
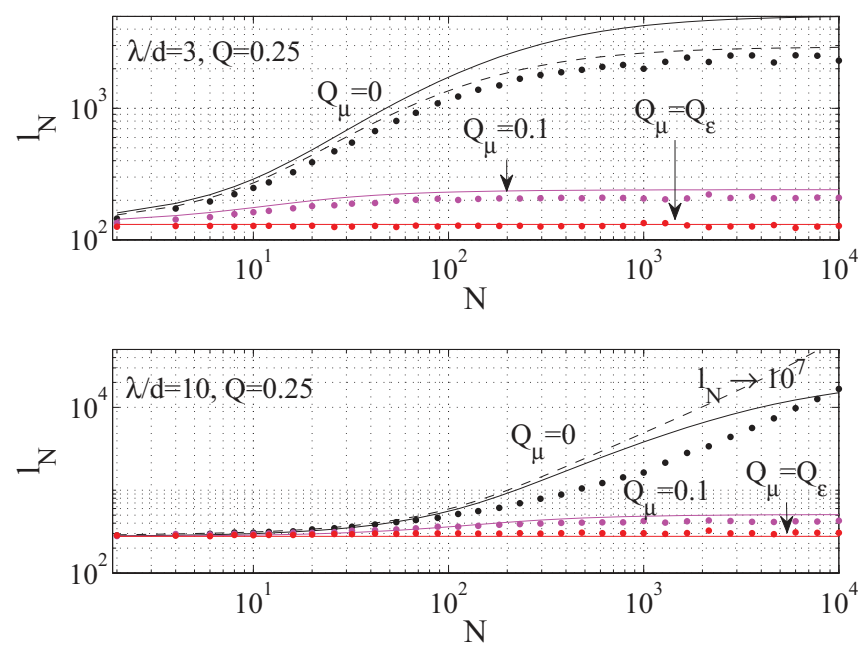

FIG. 4. (Color online) Attenuation length $l_{N}$ of a mixed stack as a function of $N$ for $\lambda / d=3$ and $\lambda / d=10$. Same convention as in Fig. 3 is used. The transition starts for $N=1 / Q^{2}(k d)^{2}(N \sim 4$ for $\lambda / d=3$ and $N \sim 40$ for $\lambda / d=10$ ). For $\lambda / d=10, Q_{\mu}=0$, the asymptotic $l_{N}=L_{\mathrm{loc}} \sim 10^{7}$ is found from Refs. 6 and 7 for $N>10^{7}$.

and 7)]. In this case, the validity of our expression in Eq. (13) is satisfactory in the whole range of frequencies.

(2) It is confirmed that, when perturbation only in $\epsilon$ is considered, the anomaly starts for $N Q^{2}(k d)^{2}=1$ (Fig. 4). For $\lambda / d \lesssim 10$, the saturation at the localization length occurs for $N Q^{2}(k d)^{2}>100$ and our calculation at second order, Eq. (9), is accurate to describe the whole transition (in this regime, the prediction of Ref. 8 does not apply). We derived the attenuation length at third order and it appears to be more accurate although still unsatisfactory for low frequencies (Fig. 4). Indeed, for $\lambda / d \gtrsim 10$, in light of the calculations in Ref. 8 , the next order in $Q^{4}$ is necessary to entirely capture the transition in the low-frequency regime and, by analyzing the numerical results in Refs. 7 and 8, it appears that the attenuation length reaches the localization length for typically $N Q^{4}(k d)^{8}>100$.

\section{CONCLUSIONS}

Our results concern the attenuation length $l_{N}$ in bilayered structures with a unit cell composed of left-handed and righthanded materials. When perturbation only in the permittivity or in the permeability is considered, our analytical expression is accurate for the structure of moderate size and for high frequencies (wavelengths of order or smaller than the unit-cell size). It must be stressed that the real left-handed layer as can be designed nowadays precisely works in this range of wavelengths and structure sizes. More importantly, our result applies for perturbation in the refractive indices resulting from perturbations in the permeability and in the permittivity, which is the case when designing left-handed material. This is of particular practical interest since we have shown that the combined effects of perturbations in permeability and permittivity strongly affect the behavior of the attenuation length and they are able to restore the ordinary Anderson localization. 


\section{ACKNOWLEDGMENTS}

We acknowledge the support from the Agence Nationale de la Recherche, ANR-10-INTB-0914 ProComedia.

\section{APPENDIX A: EXPRESSION AND ASYMPTOTICS OF THE TERM $\mathcal{A}$}

The term $\mathcal{A}$ in the expression of the attenuation length

$$
\begin{aligned}
\frac{1}{l_{N}} & =\frac{1}{l_{1}}-\frac{Q^{2}}{12} \mathcal{A}\left(k_{1} d_{1}, k_{2} d_{2}, Q, N\right)+O\left(Q^{3}\right), \\
\frac{1}{l_{1}} & =\frac{Q^{2}}{12}\left\{1-\frac{1}{2}\left[f\left(x_{1}\right) \cos 2 k_{1} d_{1}+f\left(x_{2}\right) \cos 2 k_{2} d_{2}\right]\right\},
\end{aligned}
$$

is given by

$$
\begin{aligned}
\mathcal{A}\left(\varphi_{1}, \varphi_{2}, Q, N\right)= & -\operatorname{Re}\left\{\left[e^{-2 i \varphi_{1}} X_{2} h^{2}\left(x_{1}\right)+e^{-2 i \varphi_{2}} X_{1} h^{2}\left(x_{2}\right)\right] \sigma\right. \\
& \left.+h\left(x_{1}\right) h\left(x_{2}\right)\left[2 \sigma+\sigma_{0}\right]\right\}
\end{aligned}
$$

with $\quad X_{a} \equiv \operatorname{sinc} x_{a} \quad\left(x_{a} \equiv 2 Q \varphi_{a}\right), \quad \varphi_{a} \equiv k_{a} d_{a} \quad(a=1,2)$, $X^{2} \equiv X_{1} X_{2}, \varphi \equiv \varphi_{1}+\varphi_{2}$, and $\sigma_{0} \equiv\left[1-\left(X e^{-i \varphi}\right)^{N}\right] /[1-$ $\left.\left(X e^{-i \varphi}\right)^{2}\right] e^{-2 i \varphi} / N, \sigma \equiv\left[e^{-2 i \varphi} / 2-\sigma_{0}\right] /\left[1-\left(X e^{-i \varphi}\right)^{2}\right]$.

If $\mathcal{A}$ is $O(Q)$ or of higher order, its contribution to $1 / l_{N}$ is negligible (and not pertinent) and we get $1 / l_{N} \sim 1 / l_{1}$ for all $N$ and $k d$ values. On the contrary, if $\mathcal{A}$ is of order unity, its contribution has to be accounted for. We show below that the asymptotic $N \rightarrow \infty$, in the low-frequency regime, leads to $\mathcal{A}=O\left(Q^{2}\right)$ for $\varphi_{1}+\varphi_{2} \neq 0$. When $\varphi_{1}+\varphi_{2}=0, \mathcal{A}$ is of order unity, and besides, it compensates the $1 / l_{1} \rightarrow Q^{2} / 6 \sin ^{2} k d$, which means that $1 / l_{N}$ is at least $O\left(Q^{3}\right)$ [Appendix B shows that this third order also vanishes for $N \rightarrow \infty$ in the lowfrequency regime, in agreement with the $O\left(Q^{4}\right)$ prediction given in Ref. 8].

Obviously, the asymptotic $N \rightarrow \infty$ gives

$$
\begin{aligned}
\mathcal{A}\left(\varphi_{1}, \varphi_{2}, Q, N \rightarrow \infty\right) & \\
= & -\operatorname{Re}\left\{\left[e^{-2 i \varphi_{1}} X_{2} h^{2}\left(x_{1}\right)+e^{-2 i \varphi_{2}} X_{1} h^{2}\left(x_{2}\right)\right.\right. \\
\quad & \left.\left.+2 h\left(x_{1}\right) h\left(x_{2}\right)\right]\right\} \sigma .
\end{aligned}
$$

In the low-frequency regime or small enough $Q$ value, namely $Q \varphi_{1}, Q \varphi_{2} \ll 1$, one gets

$$
\mathcal{A}\left(\varphi_{1}, \varphi_{2}, Q, N \rightarrow \infty\right)=-\frac{2 Q^{2}}{3} \operatorname{Re}\left\{\frac{\varphi_{1}^{2} e^{-2 i \varphi_{1}}+\varphi_{2}^{2} e^{-2 i \varphi_{2}}+2 \varphi_{1} \varphi_{2}+O\left[(Q \varphi)^{4}\right]}{1-e^{-2 i \varphi}+2 Q^{2} / 3\left(\varphi_{1}^{2}+\varphi_{2}^{2}\right) e^{-2 i \varphi}+O\left[(Q \varphi)^{4}\right]}\right\}
$$

In general, this term is $O\left(Q^{2}\right)$. However, if $\varphi=0$, with $\varphi_{2}=-\varphi_{1}$, we find

$$
\mathcal{A}\left(\varphi_{1},-\varphi_{1}, Q, N \rightarrow \infty\right)=2 \sin ^{2} k d+O\left[(Q \varphi)^{2}\right],
$$

which leads to $1 / l_{N}=o\left(Q^{2}\right)$ in (A1).

\section{APPENDIX B: THIRD-ORDER CALCULATION}

The third iteration consists in calculating the average of $S_{3}$, corresponding to an extra term $O\left(Q^{3}\right)$ in (4):

$$
T_{N} e^{i \phi_{N+1}}=1+S_{1}+S_{2}+S_{3}+O\left(Q^{4}\right),
$$

and we have

$$
\begin{aligned}
S_{3} \equiv & -\sum_{n=2}^{N+2} \sum_{j=2}^{n-1} \sum_{m=j}^{N+2} \delta_{n} \delta_{m} \delta_{m} e^{i\left(\phi_{j-1}-\phi_{m-1}\right)} \\
& -\sum_{n=2}^{N+2} \sum_{j=n}^{N+2} \sum_{m=2}^{j-1} \delta_{n} \delta_{m} \delta_{m} e^{i\left(\phi_{n-1}-\phi_{j-1}\right)}- \\
& -\sum_{n=2}^{N+2} \sum_{j=n}^{N+2} \sum_{m=j}^{N+2} \delta_{n} \delta_{m} \delta_{m} e^{i\left(\phi_{n-1}-\phi_{m-1}\right)} .
\end{aligned}
$$

This has been done for the mixed stack, with $k d=k_{1} d_{1}=$ $-k_{2} d_{2}$ and $Q=Q_{\epsilon}$, where the anomaly occurs (otherwise, the second-order calculation is sufficient to describe the localization length for any frequency and $N$ value). Note also that fortunately, $\delta_{n}$ is used at first order in this case and takes the simple form $\delta_{n}=1 / 2\left[\delta \mu_{n}-\delta \mu_{n-1}\right]+O(Q)$. The attenuation length $1 / l_{N}$ is

$$
l_{N}^{-1}=-\frac{1}{N} \operatorname{Re}\left(\left\langle S_{1}^{(2)}+S_{2}^{(2)}\right\rangle\right)+\frac{1}{l_{N}^{(3)}}+O\left(Q^{4}\right),
$$

where $S_{1}^{(2)}$ means the expansion of $S_{1}$ at second order in $\left(\delta \mu_{n}, \delta \mu_{n-1}\right)$ and with

$$
\frac{1}{l_{N}^{(3)}}=-\frac{1}{N} \operatorname{Re}\left(\left\langle S_{2}^{(3)}+S_{1} S_{2}+S_{3}\right\rangle\right)+O\left(Q^{4}\right) .
$$

We find

$$
\begin{gathered}
\frac{1}{l_{N}}=\frac{1}{l_{1}}-\frac{Q^{2}}{12} \mathcal{A}(k d,-k d, Q, N)+\frac{1}{l_{N}^{(3)}}, \\
\frac{1}{l_{N}^{(3)}}=\frac{Q^{3}}{12 \sqrt{3}} \sin 2 k d\left[F(X, x) \frac{1-X^{N}}{N\left(1-X^{2}\right)^{2}}+\frac{G(X, x)}{\left(1-X^{2}\right)}\right],
\end{gathered}
$$

with $x \equiv 2 Q k d, X \equiv \operatorname{sinc} x$, and

$$
\begin{aligned}
& F(X, x) \equiv-h(x)\left[\left(1+X^{2}\right)-h^{2}(x)\left(1-X^{2}\right)-2 X f(x)\right], \\
& G(X, x) \equiv-X h(x) f(x)+h(x)+l(x)\left(1-X^{2}\right),
\end{aligned}
$$

where $f(x)$ and $h(x)$ have been defined previously and $l(x) \equiv$ $-9 \sqrt{3}\left[x^{2} \operatorname{sinc} x-2(\operatorname{sinc} x-\cos x)\right] / x^{3}+3 \sqrt{3} \cos x / x$.

In general, the term is $O\left(Q^{3}\right)$ and its contribution to $1 / l_{N}$ is negligible compared to the second order in $O\left(Q^{2}\right)$. When the anomaly occurs (large $N$ and low-frequency regime), we have seen that the second-order contribution vanishes, so we 
could expect the third order to become dominant. However, it is easy to see that the third order also vanishes in these limits and the fourth order should be inspected: Indeed, in the asymptotic $N \rightarrow \infty$, the first term of $1 / l_{N}$ in Eq. (B6) vanishes. Also, in the low-frequency regime $(x=2 Q k d \ll 1)$, we have $f(x)=1+O\left(x^{2}\right), h(x) \sim x / \sqrt{3}+O\left(x^{3}\right)$, and $l(x) \sim O(x)$ [with $X \sim 1+O\left(x^{2}\right)$ ]. Obviously, this makes the term $G(X, x) /\left(1-X^{2}\right)=O(Q k d)$ vanish at order $O(1)$. This is in agreement with the prediction done by Ref. 8 that the localization length is $O\left(Q^{4}\right)$.
${ }^{1}$ D. R. Smith, J. B. Pendry, and M. C. K. Wiltshire, Science 305, 788 (2004).

${ }^{2}$ N. Engheta and R. W. Ziolkowski, in Metamaterials: Physics and Engineering Explorations (Wiley \& Sons, New York, 2006), pp. 3-30.

${ }^{3}$ Y. Dong and X. Zhang, Phys. Lett. A 359, 542 (2006).

${ }^{4}$ I. M. Lifshits, S. A. Gredeskul, and L. A. Pastur, Introduction to the Theory of Disordered Systems (Wiley, New York, 1988).

${ }^{5}$ P. Markos and C. M. Soukoulis, Wave Propagation: From Electrons to Photonic Crystals and Left-Handed Materials (Princeton University Press, Princeton, NJ, 2008).

${ }^{6}$ A. A. Asatryan, L. C. Botten, M. A. Byrne, V. D. Freilikher, S. A. Gredeskul, I. V. Shadrivov, R. C. McPhedran, and Y. S. Kivshar, Phys. Rev. Lett. 99, 193902 (2007).
${ }^{7}$ A. A. Asatryan, S. A. Gredeskul, L. C. Botten, M. A. Byrne, V. D. Freilikher, I. V. Shadrivov, R. C. McPhedran, and Y. S. Kivshar, Phys. Rev. B 81, 075124 (2010).

${ }^{8}$ E. J. Torres-Herrera, F. M. Izrailev, and N. M. Makarov, Europhys. Lett. 98, 27003 (2012).

${ }^{9}$ D. R. Smith, W. J. Padilla, D. C. Vier, S. C. Nemat-Nasser, and S. Schultz, Phys. Rev. Lett. 84, 4184 (2000).

${ }^{10}$ J. Valentine, S. Zhang, T. Zentgraf, E. Ulin-Avila, D. A. Genov, G. Bartal, and X. Zhang, Nature (London) 455, 376 (2008).

${ }^{11}$ A. A. Asatryan, L. C. Botten, M. A. Byrne, V. D. Freilikher, S. A. Gredeskul, I. V. Shadrivov, R. C. McPhedran, and Y. S. Kivshar, Phys. Rev. B 85, 045122 (2012).

${ }^{12}$ F. M. Izrailev, N. M. Makarov, and E. J. Torres-Herrera, Physica B 405, 3022 (2010). 